\title{
Estudio multicéntrico retrospectivo de los aspectos epidemiológicos, clínicos y terapéuticos de la enfermedad de Kawasaki en niños de Panamá
}

\author{
Multicenter retrospective study about the epidemiological, clinical and therapeutic aspects \\ of Kawasaki disease in children from Panama
}

\author{
Dora Estripeaut 1,8,9, Jacqueline Levy ${ }^{1,9}$, Kathia Luciani',9, Alex Tapia ${ }^{3,9}$, Carlos Daza ${ }^{4,9}$, \\ Jorge D. Méndez-Ríos ${ }^{5}$, Adriana H Tremoulet ${ }^{6,9}$ y Rolando Ulloa-Gutierrez 7,9
}

\author{
'Servicio de Infectología, Hospital del Niño Doctor José Renán Esquivel, Panamá, Panamá \\ ¿2Servicio de Infectología, Hospital de Especialidades Pediátricas Omar Torrijos Herrera (HEPOTH) de la CSS, Panamá, Panamá. \\ ${ }^{3}$ Servicio de Reumatología, Hospital de Especialidades Pediátricas Omar Torrijos Herrera (HEPOTH) de la CSS, Panamá, Panamá. \\ ${ }^{4}$ Servicio de Infectología, Hospital Materno Infantil José Domingo de Obaldía, David, Panamá. \\ ${ }^{5}$ Sección de Genética y Citogenética, CHDrAAM, Caja de Seguro Social, Panamá, Panamá. \\ ${ }^{6}$ RadyChildren's Hospital San Diego, University of California, San Diego, San Diego, CA, Estados Unidos.

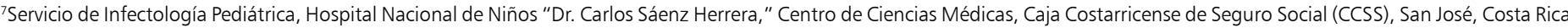 \\ ${ }^{8}$ Sistema Nacional de Investigación de SENACYT. Panamá. \\ ${ }^{9}$ Red de Enfermedad de Kawasaki en América Latina (REKAMLATINA)
}

Presentado preliminarmente como abstracto en el Congreso ID Week 2016. New Orleans, Louisiana, EEUU. Octubre 26-30, 2016.

Financiamiento: ninguno

Conflictos de interés: ninguno

Recibido: 29 de Agosto de 2020 / Aceptado: 28 de octubre de 2020

\section{Resumen}

Antecedentes: La enfermedad de Kawasaki (EK) representa la principal causa de cardiopatía pediátrica adquirida en muchos países; sin embargo, hay pocos estudios publicados en Latinoamérica. Objetivo: Describir la epidemiología, los aspectos clínicos y el tratamiento de EK en niños en Panamá como parte del estudio de vigilancia de REKAMLATINA-2 en Latinoamérica. Pacientes y Métodos: Estudio retrospectivo, descriptivo, de pacientes internados con diagnóstico de EK, atendidos en tres hospitales pediátricos de Panamá del 1-enero-2009 al 31-diciembre-2013. Resultados: Se analizaron 111 pacientes, $61(54,9 \%)$ eran hombres. Todos fueron hospitalizados, siendo la media de hospitalización de 5,8 (4-7) días. La mediana de edad al ingreso fue de 28,9 (12-38) meses. Un 63,9\% recibió antimicrobianos recientemente por otros posibles diagnósticos. Recibieron inmunoglobulina intravenosa (IGIV) $105(94,6 \%)$ pacientes; de éstos, $10(9,5 \%)$ fueron resistentes. Un 11,7\% tuvo alteraciones cardiovasculares en el ECO inicial, de las cuales las lesiones coronarias se detectaron en 3 (2,9\%) pacientes. Conclusiones: Los datos sugieren que EK en Panamá tiene una incidencia cercana a 2,05 x 100,000 bajo 15 años de edad y una frecuencia 2,6 veces mayor bajo 3 años de edad. Se observó un alto porcentaje de uso de antimicrobianos ambulatoriamente antes de la confirmación diagnóstica, lo cual sugiere reconocimiento tardío de EK en Panamá.

Palabras clave: enfermedad de Kawasaki; niños; aneurismas coronarios; Latinoamérica.

\section{Abstract}

Background: Kawasaki disease (KD) is the leading cause of pediatric acquired heart disease in many countries, however, there are few published studies from Latin America (LA). Aim: To describe the epidemiology, clinical aspects, and treatment of KD in children from Panama as part of the REKAMLATINA-2 in LA. Methods: Retrospective descriptive review of hospitalized patients diagnosed with KD, attended at three main pediatric hospitals of Panama from January-1-2009 to December-31-2013. Results: 111 patients were analyzed, 61(54.9\%) were male. All children were hospitalized, and had a mean length of hospitalization of 5.8 (4-7) days. Median age at admission was 28.9 (12-38) months. Prior to KD final diagnosis, $63.9 \%$ patients received antibiotics for other presumed diagnoses. $105(94.6 \%)$ patients received IGIV, 10 (9.5\%) were resistant. On initial echocardiogram, $11.7 \%$ of cardiovascular complications were reported, of which coronary artery lesions (CALs) were detected in $3(2.9 \%)$ patients. Conclusion: The data suggest that KD in Panama has an incidence of about $2.05 \times 100,000$ in children under 15 years of age, and with a frequency 2.6 times higher in children under 3 years. A high rate of antibiotic misuse on outpatient prior to diagnostic confirmation was observed, suggesting KD unawareness and late recognition in Panamá.

Keywords: Kawasaki disease; children; coronary aneurysms; Latin America.

\section{Correspondencia a:}

Dora Estripeaut Calderón:

destripeaut@gmail.com 


\section{Introducción}

L a enfermedad de Kawasaki (EK) es una vasculitis de etiología desconocida que conlleva a serias complicaciones cardiovasculares como aneurismas coronarias e infarto agudo de miocardio, siendo lo más frecuente la presencia de dilatación arterial. Los pacientes sin tratamiento o con demora en su diagnóstico pueden sufrir serias secuelas o muerte. Esta enfermedad fue descrita por primera vez por Tomisaku Kawasaki en 1967, demostrando una alta incidencia en la población japonesa y asiática. Se ha asociado a enfermedades infecciosas previas o a infecciones de reciente aparición por lo cual se postula un origen infeccioso y/o autoinmune, siendo la etiología multifactorial la más reconocida hasta ahora ${ }^{2}$.

La revisión más reciente de las guías diagnósticas de la Asociación Americana de Cardiología (AHA) del 2017 ha clasificado el diagnóstico de EK en clásico e incompleto ${ }^{3}$. El EK clásico consiste en la identificación de fiebre con cinco días o más de duración junto a cuatro o más de los cinco hallazgos clínicos cardinales. La segunda entidad, reconocida como EK incompleto, ha sido definida para minimizar el tiempo de diagnóstico ante cuadros sospechosos, debiéndose considerar en cualquier infante o niño con fiebre mayor a cuatro días y sin explicación, menos de cuatro de los principales hallazgos clínicos, y laboratorios alterados como hemograma completo, proteína $\mathrm{C}$ reactiva, sodio sérico, albúmina, enzimas hepáticas, así como hallazgos en el ecocardiograma ${ }^{3}$.

Dada la falta de datos epidemiológicos de esta enfermedad en América Latina, la red internacional REKAMLATINA ha lanzado estudios múlticéntricos colaborativos para evaluar el comportamiento epidemiológico de la enfermedad en nuestra región. Como parte de estos estudios, presentamos los datos obtenidos en tres centros hospitalarios de Panamá como primer esfuerzo multicéntrico para caracterizar esta enfermedad en la población pediátrica en nuestro país.

\section{Métodos}

El estudio REKAMLATINA-2 fue un estudio multicéntrico, multinacional, retrospectivo, descriptivo, de dicha red con participación de 39 hospitales en 16 países de Latinoamérica y en los cuales se enroló un total de 976 niños durante cinco años ${ }^{4}$. De esta cohorte, se realizó un sub-análisis de los casos reportados por Panamá. El presente estudio se basó en revisión de expedientes clínicos, registro de exámenes de laboratorio y estudios de gabinete de los pacientes internados con diagnóstico de EK, atendidos en tres hospitales pediátricos de la República de Panamá: Hospital del Niño Doctor José Renán Esquivel y Hospital de Especialidades Pediátricas Omar
Torrijos Herrera de la Caja del Seguro Social (ambos en Ciudad de Panamá) y Hospital Materno Infantil José Domingo De Obaldía (Chiriquí). Como parte del estudio REKAMLATINA-2, la información confidencial de los pacientes fue ingresada en el registro electrónico REDCap (Research Electronic Data Capture).

Se incluyeron los pacientes egresados con diagnóstico de EK clásico e incompleto durante el periodo del 1 de enero 2009 al 31 diciembre 2013. Los datos recolectados y depurados fueron analizados utilizando EpiInfo 7, realizando análisis de frecuencia y de dispersión para las variables recolectadas que incluyeron datos epidemiológicos, clínicos, demográficos, laboratorio, de ecografías, tratamiento efectuado, respuesta al tratamiento, y potenciales complicaciones. El análisis estadístico de las variables incluyó la determinación de frecuencia, y cálculo de medidas de resumen como cuartiles, intervalos de confianza, porcentajes y proporciones para valores nominales. Los ecocardiogramas fueron considerados anormales de existir la presencia de dilatación coronaria, aneurisma, engrosamiento de las paredes coronarias, efusión pericárdica o insuficiencia valvular. La resistencia al tratamiento fue determinada por una persistencia de temperatura corporal igual o mayor de 38 grados posterior a las 36 horas de completar el tratamiento con inmunoglobulina intravenosa (IGIV).

El estudio REKAMLATINA-2 fue aprobado inicialmente por el Comité Ético Científico del Hospital Nacional de Niños "Dr. Carlos Sáenz Herrera" en San José, Costa Rica y perteneciente a la Caja Costarricense de Seguro Social (CCSS). Así mismo, fue aprobado por el Comité de Bioética e Investigación de cada uno de los tres hospitales participantes de Panamá.

\section{Resultados}

Se incluyó para el presente análisis un total de 111 casos de EK, siendo $61(54,9 \%)$ de estos pacientes del sexo masculino (Tabla 1). La totalidad de los casos presentó un promedio de estancia hospitalaria de 5,8 días y de edad al momento de admisión de 28,9 meses. Previo al diagnóstico de EK, 63,9\% de los pacientes recibió antimicrobianos de forma ambulatoria por otros posibles diagnósticos. Tomando en cuenta los cinco años de recolección de datos de pacientes con EK (Figura 1), obtuvimos un promedio de 22 pacientes por año, y una incidencia aproximada de 2,05 x 100.000 en personas bajo 15 años de edad, de la población panameña existente durante los años de recolección de datos ${ }^{5}$.

La frecuencia de EK por grupo de edad al momento del diagnóstico se presenta en la Figura 2, con un total de 80 pacientes $(72,1 \%$ - $95 \%$ IC $62,1-79,8)$ en el grupo de edad bajo 3 años de edad. Se observó un predominio 


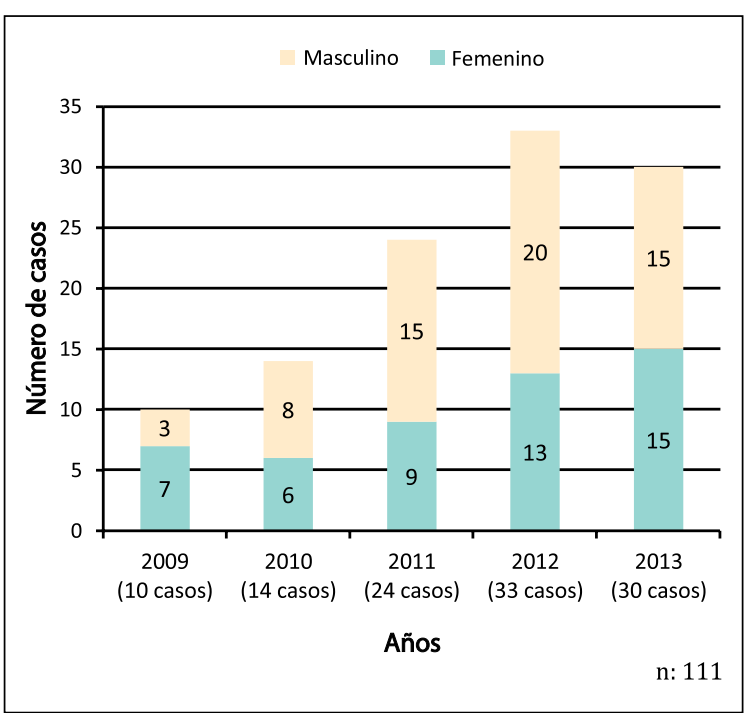

Figura 1. Distribución de casos de enfermedad de Kawasaki por año y por sexo en el período del 2009 al 2013. Panamá.

(36,0\% del total) en el segundo año de vida. Al analizar la distribución de casos por grupo de edad, se observó una frecuencia de casos de EK 2,6 veces más alta bajo 3 años de edad y un predominio de casos masculinos para ambos grupos (Tabla 2).

Al evaluar la distribución mensual de casos de EK a través de los cinco años del estudio, se observó una distribución con predominio en los últimos meses del año, entre septiembre y diciembre, con $42,3 \%$ de los casos concentrados en este periodo. Esto se contrasta con el 26,1 y $31,5 \%$ de casos diagnosticados en los periodos de enero-abril y mayo-agosto, respectivamente (Figura suplementaria 1).

Tabla 2. Distribución por sexo de los casos de enfermedad de Kawasaki bajo y sobre 3 años de edad. Panamá 2009-2013

$\begin{array}{cccc}\text { Edad/Sexo } & \text { Frecuencia } & \% & \mathbf{9 5 \%} \text { IC } \\ <3 \text { años } & 80 & 72,0 \% & 62,7-80,1 \% \\ \text { Masculino } & 43 & 53,7 \% & \\ \text { Femenino } & 37 & 46,2 \% & \\ >=3 \text { años } & 31 & 27,9 \% & 19,8-37,2 \% \\ \text { Masculino } & 18 & 58,0 \% & \\ \text { Femenino } & 13 & 41,9 \% & \\ \text { Total } & 111 & 100 \% & \end{array}$

Tabla 1. Características generales de los casos de enfermedad de Kawasaki evaluados. Panamá 2009-2013

\begin{tabular}{ll}
\hline Características & Promedio \\
\hline Número de casos & 111 \\
\hline Edad (meses) & $28,9(12-38)^{*}$ \\
\hline Días febriles previos al diagnóstico & $7,04(4-8)$ \\
\hline Varones, n (\%) & $61(54,9 \%)$ \\
\hline Días de hospitalización & $5,8(4-7) *$ \\
\hline Uso de antimicrobianos, $n$ (\%) & $69(63,8 \%)$ \\
\hline Uso de IGIV, n (\%) & $105(94,5 \%)$ \\
\hline Respuesta al tratamiento, n (\%, 95\% IC) & $90(81,0 \% ; 72,5-87,8 \%)$ \\
\hline Sensible & $13(11,7 \% ; 6,3-19,1 \%)$ \\
\hline Resistente & $8(7,2 \% ; 3,1-13,7 \%)$ \\
\hline Desconocido & $10(9,5 \% ; 4,6-16,8 \%)$, \\
\hline No disponible & $1(0,9 \% ; 0,0-5,1 \%)$ \\
\hline
\end{tabular}

*Representan los valores en los percentiles $25 \%$ y $95 \%$ respectivamente. IGIV: inmunoglobulina intravenosa.

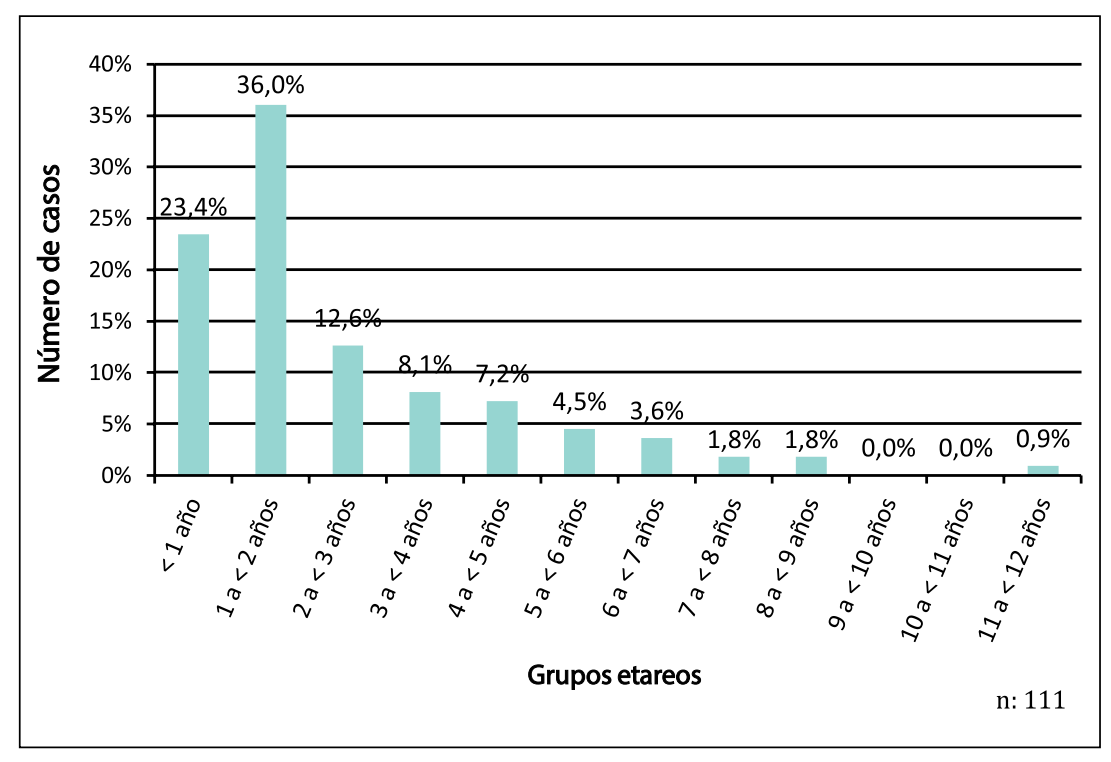

Figura 2. Distribución de casos de enfermedad de Kawasaki por grupo de edad. Panamá 2009-2013. 


\begin{tabular}{|c|c|c|c|}
\hline Hallazgos clínicos & n & $\%$ & $95 \%$ IC \\
\hline Inyección conjuntival & 99 & $89,1 \%$ & $81,8-94,2 \%$ \\
\hline Mucositis, queilitis, glositis & 91 & $81,9 \%$ & $73,5-88,6 \%$ \\
\hline Exantema cutáneo & 91 & $81,9 \%$ & $73,5-88,6 \%$ \\
\hline Edema, eritema, y/o Induración en manos o pies & 82 & $73,8 \%$ & $64,6-81,7 \%$ \\
\hline Linfadenopatía cervical & 53 & $47,7 \%$ & $38,1-57,4 \%$ \\
\hline Lengua aframbuesada & 48 & $43,2 \%$ & $33,8-52,9 \%$ \\
\hline Irritable & 44 & $39,6 \%$ & $30,4-49,3 \%$ \\
\hline Exantema o descamación en área genital o glútea & 36 & $32,4 \%$ & $23,8-41,9 \%$ \\
\hline Eritema faríngeo & 30 & $27,0 \%$ & $19,0-36,2 \%$ \\
\hline Vómitos & 20 & $18,0 \%$ & $11,3-26,4 \%$ \\
\hline Diarrea & 20 & $18,0 \%$ & $11,3-26,4 \%$ \\
\hline Tos & 17 & $15,3 \%$ & $9,1-23,3 \%$ \\
\hline Apariencia aguda de enfermedad & 9 & $8,1 \%$ & $3,7-14,8 \%$ \\
\hline Dolor abdominal & 8 & $7,2 \%$ & $3,1-13,7 \%$ \\
\hline Artralgias o dolor en extremidades & 5 & $4,5 \%$ & $1,4-10,2 \%$ \\
\hline Cambios en cicatriz de BCG & 3 & $2,7 \%$ & $0,5-7,7 \%$ \\
\hline Ictericia & 1 & $0,9 \%$ & $0,0-4,9 \%$ \\
\hline Dificultad respiratoria & 1 & $0,9 \%$ & $0,0-4,9 \%$ \\
\hline Mala perfusión distal & 0 & $0,0 \%$ & - \\
\hline Signos meníngeos & 0 & $0,0 \%$ & - \\
\hline
\end{tabular}

\begin{tabular}{|c|c|c|c|}
\hline Diagnósticos asociados & Frecuencia & $\%$ & $95 \%$ IC \\
\hline Enfermedad de Kawasaki & 80 & $72,0 \%$ & $62,7-80,1 \%$ \\
\hline Virosis & 11 & $9,9 \%$ & $5,0-17,0 \%$ \\
\hline Escarlatina & 8 & $7,2 \%$ & $3,1-13,7 \%$ \\
\hline Sepsis/shock & 4 & $3,6 \%$ & $0,9-8,9 \%$ \\
\hline Fiebre & 4 & $3,6 \%$ & $0,9-8,9 \%$ \\
\hline Síndrome de piel escaldada & 3 & $2,7 \%$ & $0,5-7,7 \%$ \\
\hline Infección urinaria & 3 & $2,7 \%$ & $0,5-7,7 \%$ \\
\hline Bacteriemia oculta & 3 & $2,7 \%$ & $0,5-7,7 \%$ \\
\hline Eritema multiforme/síndrome de Stevens-Johnson & 2 & $1,8 \%$ & $0,2-6,3 \%$ \\
\hline Enterovirosis & 2 & $1,8 \%$ & $0,2-6,3 \%$ \\
\hline Dengue & 1 & $0,9 \%$ & $0,0-4,9 \%$ \\
\hline Adenovirosis & 0 & $0,0 \%$ & - \\
\hline
\end{tabular}

Entre las manifestaciones clínicas que se reportaron en más de $50 \%$ de los casos tenemos la inyección conjuntival $(89,1 \%)$, las lesiones orales (mucositis, queilitis y glositis-82,0\%) y el exantema $(89,2 \%)$. Otros signos como edema, eritema y/o induración de manos y pies se presentaron en $73,7 \%$ de los casos. Las otras manifestaciones descritas en la literatura médica, que acompañan a la EK, se presentan en la Tabla 3.

En 72,1\% el diagnóstico de EK fue planteado al ingreso, seguido de sospecha de infecciones virales $(9,9 \%)$ y fiebre escarlatina (7,2\%). Otros diagnósticos de entrada se reportaron con menor frecuencia (Tabla 4). Los diagnósticos de ingreso ausentes en la herramienta de recolección de datos fueron registrados como "otros", y resumidos en la Tabla Suplementaria 1; entre éstos, se consignaron con relativa frecuencia manifestaciones exantemáticas tipo urticaria y cuadros de gastroenteritis y diarrea, ambos con una frecuencia de $19,0 \%$. Con menor frecuencia, 16,7 y $7,1 \%$, respectivamente, se anotaron la adenitis y la faringo-amigdalitis. Es importante destacar que estos diagnósticos fueron agrupados por sus similitudes clínicas para simplificar la presentación de los datos.

Las pruebas de laboratorio analizadas se presentan en la Tabla Suplementaria 2. A destacar tenemos que la hemoglobina promedio fue de $10,7 \mathrm{~g} / \mathrm{dL}$, hematocrito de $32,3 \%$, ambos datos compatibles con anemia. Hubo también leucocitosis y neutrofilia. Otros marcadores de procesos inflamatorios, PCR y VHS, fueron elevados. En cuanto a la química, se midió el sodio en 45 pacientes, en 18 casos (40\%) se encontró un promedio de 134,3 $\mathrm{mmol} / \mathrm{L}$.

Cerca de $59,5 \%$ de los casos correspondieron a EK clásica (95\% - IC 49,7 - 68,6\%) (Tabla 5). De este grupo, 6 casos presentaban algún hallazgo ecocardiográfico patológico, mientras que en 54 casos $(81,8 \%)$ se reportaron ecografías normales.

En cambio, 43 casos (38,7- 95\% IC 29,6-48,4\%) fueron clasificados como EK incompleto, de los cuales 34 casos $(79,1 \%)$ no mostraron patología por ecocardiografía y 7 casos $(16,3 \%)$ sí presentaron alguna alteración. En un total de 8 casos, el ecocardiograma fue inconcluso o tenía información incompleta.

En forma global, se presentaron complicaciones cardiovasculares en $11,7 \%$ de los casos.

En cuanto al abordaje terapéutico, 97,3\% (108 casos) fueron tratados con ácido acetilsalicílico (AAS) y se utilizó IGIV en 94,6\% (105 casos) de los pacientes, Del total de pacientes tratados con IGIV, en 89 casos $(80,2 \%)$ la IGIV fue administrada en los primeros 10 días de su cuadro febril (Ver Tabla 6). De los 105 pacientes que recibieron IGIV, 94 casos $(84,7 \%)$ presentaron respuesta adecuada al tratamiento, mientras que en 10 casos $(9,01 \%)$ se registró resistencia al tratamiento con persistencia de la fiebre. No hubo fallecidos en nuestro estudio. 


\begin{tabular}{|c|c|c|c|}
\hline Kawasaki clásico & Frecuencia & $\%$ & $95 \%$ IC \\
\hline Ecocardiografía normal & 54 & $81,8 \%$ & $70,3-90,2 \%$ \\
\hline Ecocardiografía patológica & 6 & $9,0 \%$ & $3,4-18,7 \%$ \\
\hline $\begin{array}{c}\text { Insuficiencia valvular } \\
\text { Efusión pericárdica }\end{array}$ & & & \\
\hline No especificado & & & \\
\hline No realizado o inconclusa & 6 & $9,0 \%$ & $3,4-18,7 \%$ \\
\hline Total & 66 & $100 \%$ & \\
\hline Kawasaki incompleto & Frecuencia & $\%$ & $95 \%$ IC \\
\hline Ecocardiografía normal & 34 & $79,0 \%$ & $63,9-89,9 \%$ \\
\hline $\begin{array}{c}\text { Ecocardiografía patológica } \\
\text { Dilatación arterial } \\
\text { Efusión pericárdica }\end{array}$ & 7 & $16,2 \%$ & $6,8-30,7 \%$ \\
\hline $\begin{array}{r}\text { Estenosis pulmonar } \\
\text { No especificado }\end{array}$ & & & \\
\hline No realizado o inconclusa & 2 & $4,6 \%$ & $0,5-15,8 \%$ \\
\hline Total & 43 & $100 \%$ & \\
\hline Kawasaki no clasificado & Frecuencia & $\%$ & $95 \%$ IC \\
\hline Ecocardiografía normal & 2 & $100 \%$ & $15,8-100 \%$ \\
\hline Total & 2 & $100 \%$ & \\
\hline
\end{tabular}

\begin{tabular}{|c|c|c|c|}
\hline Respuesta a tratamiento & Frecuencia & $\%$ & $95 \%$ IC \\
\hline Adecuada & 94 & $84,6 \%$ & $76,6-90,8 \%$ \\
\hline Resistencia & 10 & $9,0 \%$ & $4,4-15,9 \%$ \\
\hline No consignada & 7 & $6,3 \%$ & $2,5-12,5 \%$ \\
\hline Total & 111 & $100 \%$ & \\
\hline
\end{tabular}

\section{Discusión}

La EK es una vasculitis de gran importancia en pediatría por la dificultad de su diagnóstico y la necesidad de iniciar tempranamente su tratamiento para evitar serias complicaciones cardiovasculares. La falta de estudios de esta enfermedad en Latinoamérica y Panamá limita el conocimiento que se tiene de ella y el comportamiento en nuestra población. Los resultados de este estudio arrojan que la EK en Panamá se comporta de forma similar en varios aspectos a lo ya reportado en otras latitudes, como la edad promedio de 29 meses, la que es similar a lo reportado en poblaciones latinoamericanas ${ }^{6}$. También se evidenció una frecuencia 2,6 veces mayor en pacientes bajo 3 años de edad y en forma similar con lo encontrado en otros países, se observó un predominio del sexo masculino independientemente de la edad ${ }^{6,7}$.
El hallazgo de aumento de frecuencia en los meses de estación lluviosa en el Panamá (septiembre a diciembre) coincide con los incrementos de cuadros infecciosos virales en niños. Esto refuerza la hipótesis del origen o gatillo infeccioso de la enfermedad.

Llama la atención que $72 \%$ de estos casos fueron identificados a su ingreso como EK, dando confianza en el reconocimiento y sospecha clínica, al menos en nuestra institución hospitalaria. Sin embargo, como hospital de referencia algunos de estos pacientes ya venían referidos con diagnóstico de EK. En esta serie, las manifestaciones clínicas clásicas de EK incluyeron mucositis, queilitis, glositis, exantemas, edema y eritemas de manos y pies, linfadenopatías, y lengua "aframbuesada".

Diagnósticos de ingreso alternativos incluyeron sospechas de infección viral y fiebre escarlatina, ambos con parcial similitud a la EK. Otros diagnósticos adicionales anotados, como exantemas inespecíficos, manifestaciones gastrointestinales, adenitis y faringoamigdalitis, también coinciden con hallazgos clínicos frecuentes en la EK. Sin embargo, llama la atención el alto porcentaje de pacientes que recibió antimicrobianos de manera ambulatoria antes de su ingreso hospitalario, lo que sugiere que el diagnóstico aún no se sospecha oportunamente. Estos porcentajes coincide con otros reportes en América Latina de nuestra red donde como parte del estudio paralelo prospectivo REKAMLATINA-1; encontramos que, en Costa Rica, México y otros países de la región el uso previo de antimicrobianos ocurrió en 82 - 78 y $74 \%$, respectivamente ${ }^{8-10}$.

Los hallazgos de laboratorio también fueron similares a lo descrito en otras poblaciones en quienes se ha demostrado cuadros de anemia $^{11,12}$, leucocitosis y neutrofilia, junto a la elevación de la PCR y VHS. El análisis de las variables de laboratorio no demostró un incremento clínicamente significativo para predecir complicaciones cardiovasculares o la gravedad del cuadro, o resistencia al tratamiento a diferencia de lo demostrado por otros grupos ${ }^{13}$.

Dentro de los 111 pacientes incluidos en el análisis, a 59,5\% se le catalogó como EK clásico y el restante $38,7 \%$ como EK incompleto. Este porcentaje de casos clásicos son más elevado que el $15 \%$ documentado por otros grupos asiáticos ${ }^{7}$, lo cual debe verificarse en estudios prospectivos.

Las complicaciones cardiovasculares se presentaron en $11,7 \%$ de los casos y no se observó diferencia significativa al estratificarlo por el tipo de EK. De rescatar, la gran mayoría de los casos $(81,1 \%)$ no presentó complicaciones cardiovasculares independientemente del tipo de EK. La frecuencia cercana a $10 \%$ de complicaciones cardiovasculares es más baja al compararla con reportes de India ${ }^{14}$, y similar al 8,3\% de los casos reportados en Japón ${ }^{13}$.

El tratamiento de estos pacientes consistió en el uso de IGIV y AAS. De los 111 pacientes que recibieron IGIV, se documentó una buena respuesta al tratamiento en 94 casos, y aparición de resistencia al tratamiento en $10(9 \%)$ casos, muy similar a la resistencia de 10 a $20 \%$ recientemente publicada por la Asociación Americana del Corazón y otros grupos ${ }^{2,49,10}$. En una revisión de expedientes realizada en el Hospital del Niño de Panamá en 2015, se encontró $8,7 \%$ de resistencia ${ }^{15,16}$.

Dada la frecuencia determinada de 22 pacientes por año en promedio dentro del período observado de 2009 al 2013, y tomando en cuenta un estimado de la población infantil, se obtuvo una incidencia aproximado de 2,05 x 100,000 en pacientes bajo 15 años de edad. Esta incidencia es 
muy similar a la reportada en poblaciones europeas, y de 100 a 180 veces más bajas al compararlo con la incidencia en algunas regiones de $\mathrm{Asia}^{7,17}$. Aunque reconocemos que se deben realizar estudios en muestras más representativas de la población general, se ha obtenido por primera vez en Panamá una aproximación a la incidencia anual que sirve de referencia para la toma de decisiones en salud pública y base para próximas investigaciones. En Latinoamérica pocos países han logrado la determinación de la incidencia global, mas podemos mencionar a Chile con reportes de incidencia en múltiples regiones del país ${ }^{18,19}$.

Reconocemos las limitaciones y fortalezas de nuestro estudio. En cuanto a las primeras, destacan las propias de cualquier estudio retrospectivo, tal y como la ausencia parcial de información de interés, en especial al momento de su ingreso. También, debemos mencionar que únicamente logramos captar aquellos pacientes con fiebre persistente a pesar del tratamiento dado a nivel primario. Tercero, por la naturaleza retrospectiva del estudio, no se contó con información completa de todos los ecocardiogramas en las citas de seguimiento debido a que un grupo de pacientes se perdió de control o siguió control en otro centro. En cuanto a las fortalezas, consideramos que la muestra fue bastante representativa de la población en el país, dado que se obtuvieron casos de varios centros hospitalarios, y donde se atiende la mayoría de pacientes del país. También se destaca el hecho de que es un estudio multicéntrico y es la primera vez en Panamá que se realiza para EK. A su vez, estos son los datos locales más actualizados sobre EK que se publican, existiendo solamente dos publicaciones previas de EK en nuestro país, y un reciente análisis prospectivo como parte del estudio REKAMLATINA-1 que incluye datos de junio del 2014 a marzo del $2017^{20}$. Finalmente, la presente casuística representa la serie más grande de EK de Centroamérica y el Caribe publicada en la literatura indexada en PubMed.

\section{Conclusiones}

La EK es una vasculitis con riesgo de presentar complicaciones cardiovasculares serias, predominantemente en niños.

Los datos sugieren que el EK en Panamá tiene una incidencia cercana a 2,05 x 100,000 en pacientes bajo 15 años de edad, y con una frecuencia 2,6 veces más alta bajo 3 años. Se observó un predominio en varones y siendo la EK clásica la forma de presentación predominante, descrita en $81,5 \%$ de los casos. También se observó una alta frecuencia en el uso de antimicrobianos de manera ambulatoria antes de la confirmación diagnóstica en estos pacientes.

La mayoría de los casos fueron identificados como EK correctamente en $72,1 \%$, y en su mayoría, presentando de 4 a 8 días febriles previos al diagnóstico. Las complicaciones cardiovasculares fueron identificadas por ecocardiograma en $11,7 \%$. La respuesta a IGIV fue adecuada en su mayoría, con una resistencia al tratamiento inicial que se manifestó en $10 \%$ aproximadamente.

Agradecimientos. Extendemos nuestra gratitud al sistema de registro electrónico REDCap (Research Electronic Data Capture), gracias al cual se logró ingresar la información de cada uno de los pacientes. (https://www.project-redcap.org).
Figura Suplementaria 1. Frecuencia de casos de enfermedad de Kawasaki acumulados por mes. Panamá: 2009 al 2013

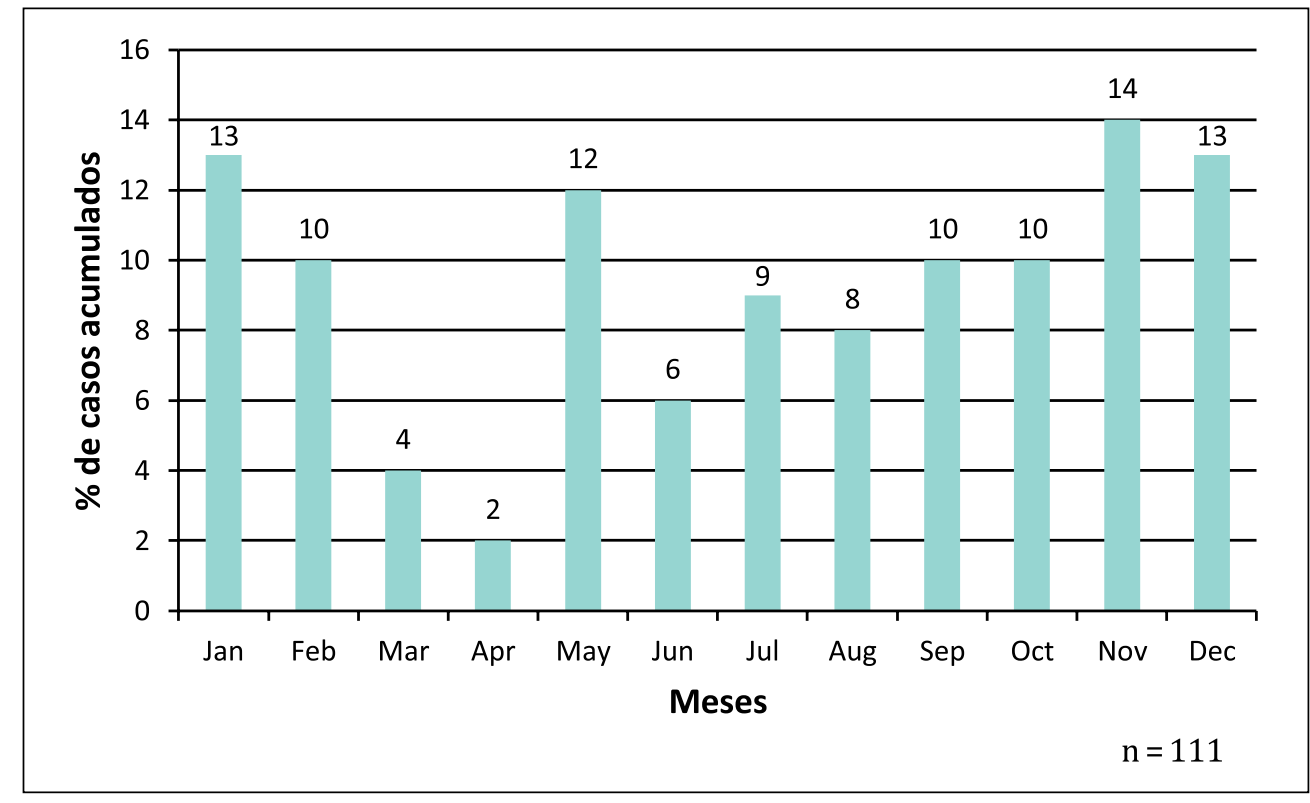




\begin{tabular}{|lccc|}
\hline $\begin{array}{l}\text { Tabla Suplementaria 1. Frecuencia de diagnósticos adicionales en } \\
\text { pacientes con enfermedad de Kawasaki. Panamá }\end{array}$ & \multicolumn{2}{l}{ 2009-2013 } \\
\hline Otros Diagnósticos asociados & Frecuencia & $\%$ & $95 \%$ IC \\
\hline Exantema/urticaria/alergia & 8 & $19,0 \%$ & $8,6-34,1 \%$ \\
\hline Gastroenteritis aguda/diarrea & 8 & $19,0 \%$ & $8,6-34,1 \%$ \\
\hline Adenitis cervical o maxilar & 7 & $16,6 \%$ & $6,9-31,3 \%$ \\
\hline Infección de vías superiores & 3 & $7,1 \%$ & $1,5-19,4 \%$ \\
\hline Neumonía & 3 & $7,1 \%$ & $1,5-19,4 \%$ \\
\hline Anemia & 2 & $4,7 \%$ & $0,5-16,1 \%$ \\
\hline Conjuntivitis & 2 & $4,7 \%$ & $0,5-16,1 \%$ \\
\hline Dactilitis & 2 & $4,7 \%$ & $0,5-16,1 \%$ \\
\hline Artritis reactiva & 1 & $2,3 \%$ & $0,0-12,5 \%$ \\
\hline Deshidratación & 1 & $2,3 \%$ & $0,0-12,5 \%$ \\
\hline Eritrodermia & 1 & $2,3 \%$ & $0,0-12,5 \%$ \\
\hline Gingivoestomatitis & 1 & $2,3 \%$ & $0,0-12,5 \%$ \\
\hline Infección de vías urinarias & 1 & $2,3 \%$ & $0,0-12,5 \%$ \\
\hline Meningitis & 1 & $2,3 \%$ & $0,0-12,5 \%$ \\
\hline Otitis media aguda & $2,3 \%$ & $0,0-12,5 \%$ \\
\hline
\end{tabular}

\begin{tabular}{|c|c|}
\hline Prueba & Promedio $\pm \mathrm{DE}$ \\
\hline Hemoglobina (n: 109) & $10,7 \mathrm{~g} / \mathrm{dL} \pm 1,1 \mathrm{~g} / \mathrm{dL}$ \\
\hline Hematocrito (n: 109) & $32,2 \% \pm 3,1 \%$ \\
\hline Plaquetas (n: 108) & $399,3 / \mathrm{mm}^{3} \pm 169,6 / \mathrm{mm}^{3}$ \\
\hline Leucocitos (n: 108) & $15,49 \times 10^{3} / \mathrm{mm}^{3} \pm 5.61 \times 10^{3} / \mathrm{mm}^{3}$ \\
\hline Segmentados (n: 100) & $9,26 \times 10^{3} / \mathrm{mm}^{3} \pm 4.67 \times 10^{3} / \mathrm{mm}^{3}$ \\
\hline Linfocitos (n: 91) & $3,74 \times 10^{3} / \mathrm{mm}^{3} \pm 2.27 \times 10^{3} / \mathrm{mm}^{3}$ \\
\hline Monocitos (n: 96) & $1,02 \times 10^{3} / \mathrm{mm}^{3} \pm 1.3 \times 10^{3} / \mathrm{mm}^{3}$ \\
\hline Bandas (n: 49) & $1,6 \times 10^{3} / \mathrm{mm}^{3} \pm 1.72 \times 10^{3} / \mathrm{mm}^{3}$ \\
\hline Eosinófilos ( $\mathrm{n}: 80$ ) & $0,64 \times 10^{3} / \mathrm{mm}^{3} \pm 1.11 \times 10^{3} / \mathrm{mm}^{3}$ \\
\hline Proteína C reactiva (n: 75) & $72,9 \mathrm{mg} / \mathrm{L} \pm 57,7 \mathrm{mg} / \mathrm{L}$ \\
\hline VHS (n: 90) & $47,4 \mathrm{~mm} / \mathrm{hr} \pm 16 \mathrm{~mm} / \mathrm{hr}$ \\
\hline Sodio (n: 40) & $134,3 \mathrm{mmol} / \mathrm{L} \pm 3,1 \mathrm{mmol} / \mathrm{L}$ \\
\hline Proteínas totales (n: 5) & $6,9 \mathrm{~g} / \mathrm{dL} \pm 1,3 \mathrm{~g} / \mathrm{dL}$ \\
\hline Albúmina (n: 38) & $3,5 \mathrm{~g} / \mathrm{dL} \pm 0,4 \mathrm{~g} / \mathrm{dL}$ \\
\hline Globulinas (n: 3) & $2,9 \mathrm{~g} / \mathrm{dL} \pm 0,2 \mathrm{~g} / \mathrm{dL}$ \\
\hline $\operatorname{ALT}(\mathrm{n}: 72)$ & $73,5 \mathrm{U} / \mathrm{L} \pm 80,0 \mathrm{U} / \mathrm{L}$ \\
\hline $\operatorname{AST}(n: 72)$ & $53,9 \mathrm{U} / \mathrm{L} \pm 41,8 \mathrm{U} / \mathrm{L}$ \\
\hline GGT (n: 12) & $106,6 \mathrm{U} / \mathrm{L} \pm 128,1 \mathrm{U} / \mathrm{L}$ \\
\hline Bilirrubina total ( $n: 47)$ & $0,5 \mathrm{mg} / \mathrm{dL} \pm 0,7 \mathrm{mg} / \mathrm{dL}$ \\
\hline Bilirrubina directa (n: 43) & $0,2 \mathrm{mg} / \mathrm{dL} \pm 0,5 \mathrm{mg} / \mathrm{dL}$ \\
\hline Bilirrubina indirecta ( $\mathrm{n}: 43$ ) & $0,3 \mathrm{mg} / \mathrm{dL} \pm 0,3 \mathrm{mg} / \mathrm{dL}$ \\
\hline
\end{tabular}

\section{Referencias bibliográficas}

1.- Estripeaut D, Levy J, Luciani K, Daza C, Salgado AP, Tremoulet AH, Ulloa-Gutierrez R. Multicenter study of the epidemiology, clinical aspects and treatment of Kawasaki disease in children from Panama: a 5-year retrospective study. En: ID Week 2016. New Orleans, Louisiana, EEUU. October 26-30, 2016. Poster 2263.

2.- Burns JC, Kushner HI, Bastian JF, Shike H, Shimizu C, Matsubara T, et al. Kawasaki disease: a brief history. Pediatrics 2000: 106, e27-e27. doi: 10.1542/peds.106.2.e27.

3.- McCrindle B, Rowley A, Newburger J, Burns J, Bolger A, Gewitz M, et al. Diagnosis, treatment, and long-term management of Kawasaki disease: a scientific statement for health professionals from the American Heart Association. Circulation 2017; 135, e927-e999m doi: 10.1161/ CIR.0000000000000484.

4.- Ulloa-Gutierrez R, Salgado AP, GarridoGarcía LM, Saltigeral-Simental P, Dueñas L, Estripeaut D, et al. Epidemiological, clinical aspects and treatment of Kawasaki disease among 976 children from 16 Latin American countries: a 5-year retrospective multinational study of the REKAMLATINA network (January 2009-December 2013). En: The $12^{\text {th }}$ International Kawasaki Disease Symposium. Yokohama, Japan. June 12-15, 2018. Poster 31.

5.- $\quad$ OPS. Visualización de indicadores. 2018 Diciembre; Available from: https://www.paho. org/data/index.php/es/?option=com_content $\&$ view $=$ article \&id=515:indicadoresviz\&Item id $=347$.

6.- García Rodríguez F, Flores Pineda AJ, Villarreal Treviño AV, Salinas Encinas DR, Lara Herrera PB, Maldonado Velázquez MDR, et al. Enfermedad de Kawasaki en un hospital pediátrico en México. Bol Méd Hosp Infant México 2016; 73: 166-73. doi:10.1016/j. bmhimx.2016.01.002

7.- Nakamura Y, Yashiro M, Uehara R, Sadakane A, Tsuboi S, Aoyama Y, et al. Epidemiologic features of Kawasaki disease in Japan: Results of the 2009-2010 nationwide survey. J Epidemiol 2012: 22: 216-21. doi: 10.2188/jea. JE20110126.

8.- Ulloa-Gutierrez R, Salgado AP, Dueñas L, Garrido-García LM, Estripeaut D, Faugier-
Fuentes E, et al. Antibiotic use and number of medical visits prior to Kawasaki disease diagnosis in Latin American children: a prospective multinational multicenter study of the REKAMLATINA network. En: $9^{\text {th }}$ World Congress of the World Society for Pediatric Infectious Diseases (WSPID). Rio de Janeiro, Brazil. November 18-21, 2015. Poster 108

9.- Ulloa-Gutierrez R, Garrido-García LM, Yamazaki-Nakashimada MA, SaltigeralSimental P, Faugier-Fuentes E, MartínezMedina L, et al. Kawasaki disease in Mexican children: results from a prospective multicentre hospital-based epidemiological and clinical study of the REKAMLATINA Network. En: $28^{\text {th }}$ International Congress of Pediatrics (IPA-2016). Vancouver, British Columbia, Canada. August 17-22, 2016. Abstract OP-44.

10.- Ulloa-Gutierrez R, Camacho-Badilla K, Soriano-Fallas A, Valverde-Muñoz K, NaranjoZuñiga G, Salgado AP, et al. Prospective study about the epidemiology, clinical presentation and treatment of Kawasaki disease in Costa Rican children. En: $28^{\text {th }}$ International Congress of Pediatrics (IPA-2016). Vancouver, British 
Columbia, Canada. August 17-22, 2016. Poster PP-115.

11.- Chaiyarak K, Durongpisitkul K, Atta T, Soongswang J, Laohaprasitiporn D, Nana A. Clinical manifestations of Kawasaki disease: what are the significant parameters? Asian Pac J. Allergy Immunol 2009; 27: 131-6. PMID: 19839499.

12.- Liu G, Wang S, Du Z. Risk factors of intravenous immunoglobulin resistance in children with Kawasaki disease: A metaanalysis of case-control studies. Front Pediatr 2020; 8: 187. doi: 10.3389/fped.2020.00187. https://doi.org/10.3389/fped.2020.00187.

13.- Goto R, Inuzuka R, Shindo T, Namai Y, Oda Y, Harita Y, et al. Relationship between post-IGIV IgG levels and clinical outcomes in Kawasaki disease patients: new insight into the mechanism of action of IGIV. Clin Rheumatol 2020. doi: 10.1007/s10067-020-05153-w.
14.- Shenoy B, Singh S, Ahmed MZ, Pal P, Balan $\mathrm{S}$, Vishwanathan $\mathrm{V}$, et al. Indian Academy of Pediatrics position paper on Kawasaki disease. Indian Pediatr 2020. S097475591600188. PMID: 32471961.

15.- Levy J, McLenan M. Factores de riesgo en pacientes con enfermedad de Kawasaki con falla al tratamiento inicial con Niño del 1 de Enero de 2003 al 31 de diciembre de 2013. Pediatr Panamá 2015; 44: 7-15. http://docs.bvsalud.org/ biblioref/2018/02/848687/20154417-15.pdf.

16.- Troitiño M., Norte G. Sáez-Llorens X. Características epidemiológicas de la enfermedad de Kawasaki en los niños panameños. Pediatr Panamá 2003; 32: 73-7.

17.- Ae R, Makino N, Kosami K, Kuwabara M, Matsubara Y, Nakamura Y. Epidemiology, treatments, and cardiac complications in patients with Kawasaki disease: The nationwide survey in Japan, 2017-2018. J Pediatr 2020; 225: 23-9.e2. doi:10.1016/j. jpeds.2020.05.034.

18.- Borzutzky A, Hoyos-Bachiloglu R, Cerda J, Talesnik E. Rising hospitalization rates of Kawasaki disease in Chile between 2001 and 2007. Rheumatol. Int. 2012; 32: 2491-5. doi: 10.1007/s00296-011-2050-4.

19.- Hoyos-Bachiloglu R, García A, Morales P, Cerda J, Talesnik E, Borzutzky A. Distribución geográfica de la enfermedad de Kawasaki en Chile. Rev. Chil. Infectol 2016; 33: 12-8. doi: 10.4067/S0716-10182016000100002.

20.- Estripeaut D, Castaño E, Norero X, Esquivel R, Levy J, Sinisterra S, et al. Estudio prospectivo multicéntrico sobre la epidemiología, presentación clínica y tratamiento de la enfermedad de Kawasaki en niños panameños. En: API 2017. Panamá, Panamá. Mayo 16-20, 2017. Poster G3. 